\title{
Colon capsule endoscopy in colorectal cancer screening: a randomised controlled trial
}

\author{
Lasse Kaalby (D) ,,2 Ulrik Deding (D) , 1,2 Morten Kobaek-Larsen (D) ,,2 \\ Anne-Line Volden Havshoi (1) ," Erik Zimmermann-Nielsen (D) , 1,2 \\ Marianne Kirstine Thygesen (D) ,,2 Rasmus Kroijer (D) ,3 \\ Thomas Bjørsum-Meyer (1) , ${ }^{1}$ Gunnar Baatrup (1) 1,2
}

To cite: Kaalby L, Deding U, Kobaek-Larsen M, et al. Colon capsule endoscopy in colorectal cancer screening: a randomised controlled trial. BMJ Open Gastro 2020;7:e000411. doi:10.1136/ bmjgast-2020-000411

Received 27 March 2020 Revised 7 May 2020 Accepted 31 May 2020

\section{Check for updates}

(c) Author(s) (or their employer(s)) 2020. Re-use permitted under CC BY-NC. No commercial re-use. See rights and permissions. Published by BMJ.

${ }^{1}$ Department of Surgery, Odense University Hospital, Svendborg, Denmark

${ }^{2}$ Department of Clinical Research, University of Southern Denmark, Odense, Denmark

${ }^{3}$ Department of Surgery, Southwest Jutland Hospital Esbjerg, Esbjerg, Denmark

Correspondence to Lasse Kaalby; Ikm@rsyd.dk

\section{ABSTRACT}

Introduction The use of capsule endoscopy has become an approved method in small bowel diagnostics, but the same level of integration is not seen in large bowel diagnostics. We will use colon capsule endoscopy (CCE) as a filter test in colorectal cancer (CRC) screening between the faecal immunochemical test (FIT) and colonoscopy. We aim to investigate the clinical performance, population acceptability, and economic implications of the procedure in a large-scale clinical trial.

Methods and analysis We will randomly allocate 124 214 Danish citizens eligible for participation in the nationa CRC screening programme within the Region of Southern Denmark to either an intervention group or a control group. Prior to submitting a FIT, citizens randomised to the intervention group will be informed about their opportunity to undergo CCE, instead of colonoscopy, if the FIT is positive. Suspected cancers; $>3$ adenomas $<10 \mathrm{~mm}$ in size, 1 adenoma $>10 \mathrm{~mm}$ in size or $>4$ adenomas regardless of size, detected during CCE will generate an invitation to colonoscopy as per regular screening guidelines, whereas citizens with suspected low risk polyps will re-enter the biennial screening programme. Citizens with no CCE findings will be excluded from screening for 8 years. In the control group, citizens will follow standard screening procedures.

Ethics and dissemination All participants must consent prior to capsule ingestion. All collected data will be handled and stored in accordance with current data protection legislation. Approvals from the regional ethics committee (ref. S-20190100) and the Danish data protection agency have been obtained (ref. 19/29858). Trial registration details The study has been registered with ClinicalTrials.gov under: NCT04049357.

\section{INTRODUCTION}

Screening for colorectal cancer (CRC) has been implemented in many countries. ${ }^{1}$ Previous studies have found that CRC screening using faecal occult blood testing reduce both short-term and long-term disease-specific mortality. ${ }^{2-6}$

During the roll-out of the Danish CRC screening programme between 2014 and
2017, some issues arose on decreasing participation, adherence to colonoscopy, colonoscopy capacity, major complications and high rates of clean colon after diagnostic colonoscopy. ${ }^{7-11}$ Several procedures addressing these issues are becoming available to healthcare providers. One is the colon capsule endoscopy (CCE), which has been found to increase screening participation, ${ }^{12}$ and which has been associated with less expected and experienced patient discomfort compared with colonoscopy. ${ }^{13}$ In addition, CCE has been found to reduce the need for colonoscopy, and may appear to be superior regarding polyp-detection rate and sensitivity to polyps $>9 \mathrm{~mm}$. ${ }^{1415}$

Introducing CCE as a filter test before colonoscopy has been investigated in a small population by Holleran et $a l,{ }^{15}$ but to the author's knowledge no large-scale multicentre trial has yet been attempted to integrate CCE in an organised screening programme.

Thus, the primary aim of this study is to introduce CCE to organised CRC screening, and to compare the number of detected cancers and intermediate-risk and high-risk adenomas between the intervention group and the control group.

\section{METHODS AND ANALYSIS}

The Danish colorectal cancer screening programme

In Denmark, the national CRC screening programme was implemented in 2014 inviting all citizens between the age of 50 and 74 biennially. ${ }^{16}$ The programme uses a stool sample, the faecal immunochemical test (FIT), to identify traces of occult blood. Citizens are invited by mail and receive a FIT-kit alongside a written instruction. Participation is accepted by submitting the faecal 
Table 1 Post-polypectomy risk-stratification for surveillance according to primary screening colonoscopy findings in the Danish colorectal cancer screening programme $^{19}$

\begin{tabular}{|c|c|c|}
\hline Classification & Findings & Surveillance \\
\hline Clean colon & No polyps & $\begin{array}{l}\text { No surveillance, } \\
\text { return to FIT after } 8 \\
\text { years }\end{array}$ \\
\hline $\begin{array}{l}\text { Low-risk } \\
\text { polyps }\end{array}$ & $\begin{array}{l}\text { No more than two } \\
\text { adenomas both }<10 \\
\mathrm{~mm} \text { in size }\end{array}$ & $\begin{array}{l}\text { No surveillance, } \\
\text { return to biennial } \\
\text { screening }\end{array}$ \\
\hline $\begin{array}{l}\text { Intermediate- } \\
\text { risk polyps }\end{array}$ & $\begin{array}{l}\text { Three to four } \\
\text { adenomas }<10 \mathrm{~mm} \text { in } \\
\text { size or one adenoma } \\
10 \text { to } 19 \mathrm{~mm} \text { in size } \\
\text { or villous histology } \\
>25 \% \text { or high-grade } \\
\text { dysplasia }\end{array}$ & $\begin{array}{l}\text { Surveillance } \\
\text { colonoscopy after } 3 \\
\text { and } 8 \text { years. }\end{array}$ \\
\hline $\begin{array}{l}\text { High-risk } \\
\text { polyps }\end{array}$ & $\begin{array}{l}\text { Greater than four } \\
\text { adenomas or one } \\
\text { adenoma } \geq 20 \mathrm{~mm} \\
\text { in size or piecemeal } \\
\text { resection of any } \\
\text { adenoma }\end{array}$ & $\begin{array}{l}\text { Surveillance } \\
\text { colonoscopy in 1, } 4 \\
\text { and } 9 \text { years. }\end{array}$ \\
\hline Cancer & Cancer suspect & $\begin{array}{l}\text { Biopsy-guided } \\
\text { immediate treatment } \\
\text { decision (surgery, } \\
\text { oncology, palliative) }\end{array}$ \\
\hline
\end{tabular}

FIT, faecal immunochemical test.

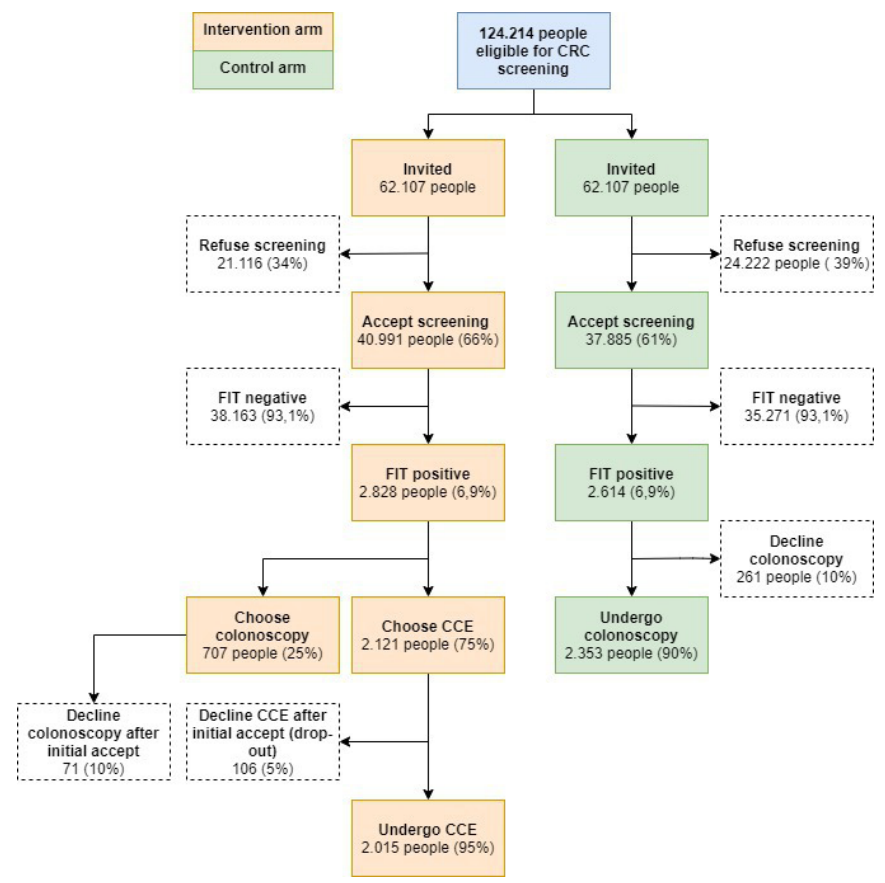

Figure 1 Expected flow in the intervention and the control group. CCE,colon capsule endoscopy; CRC, colorectal cancer;FIT, faecal immunochemicaltest. sample by mail to the hospital laboratory. Those testing FIT-positive (FIT $>100 \mu \mathrm{g} \mathrm{hgb/mL} \mathrm{buffer),} \mathrm{are} \mathrm{invited}$ to undergo colonoscopy within 14 days for further diagnostics. During the procedure, all detected polyps are removed, whereas suspected cancers are biopsied. Based on a post-polypectomy risk-stratification (table 1), participants with intermediate-risk or high-risk adenomas are offered endoscopic surveillance. Participants with lowrisk adenomas will return to the biennial FIT screening programme, whereas those with no findings (clean colon) will receive an 8-year long exclusion.

\section{Study design}

Our study is a multicentre, randomised controlled trial. The study population includes all citizens, regardless of former participation, invited for CRC screening in the Region of Southern Denmark from August 2020 and onwards. An estimated 124214 people will be randomised 1:1 to either the intervention group or the control group (figure 1) using an automated randomisation tool provided by Odense Patient data Explorative Network (OPEN). Citizens in the intervention group will be offered the choice between CCE and colonoscopy, in case of a positive FIT. The other half will follow the screening programme as usual. All FIT positive citizens in the inclusion period, including those in the control group, will be followed via national registers. The randomisation will be based on Zelen's pre-randomisation principle. $^{17}$

\section{Inclusion and invitation}

The randomisation will be managed by the Invitation and Administration Module of the screening programme. Citizens invited for screening in even weeks will be allocated to the intervention group, whereas citizens invited in odd weeks will be allocated to the control group. The effectuation of the intervention will be conducted at four selected out-clinic sites in the Region of Southern Denmark. The only eligibility criterion is participation in the Danish CRC screening programme within the Region of Southern Denmark. Eligible participants randomised to the intervention group will receive information about the study alongside their regular screening invitation, stating that in the event of a returned and positive FIT, they will be offered a CCE as the initial bowel investigation procedure as an alternative to colonoscopy. They will then have the opportunity to mark a positive interest in CCE in case of positive FIT by attaching a green sticker to the sample vial. If the invitee has no interest in undergoing CCE, a red sticker attached to the sample vial will indicate a wish to adhere to regular screening protocols. If no sticker is attached to the sample vial for participants in the intervention group, the invitee will be invited for colonoscopy in case of a positive FIT. Invitees in the intervention group will be able to change their choice of investigation throughout the process provided that they accept the delay due to changes. 
Invitees will be informed that they have the option to refuse CCE participation and undergo colonoscopy instead, or refuse further investigations altogether. Participants who refuse CCE and choose to undergo colonoscopy will remain in the study analysis, as intention-to-treat in the intervention group.

The control group will participate per regular screening guidelines, unaware of our study. The control group will follow regular screening protocols in terms of eligibility to participate.

\section{Exclusion criteria}

The exclusion criteria below are only for those randomised to the intervention group.

1. History of abdominal surgery (excluding appendectomy).

2. Symptomatic Crohn's disease.

3. Cardiac pacemaker.

4. Known renal insufficiency.

5. Pregnancy/breast feeding.

6. Allergy towards bowel preparation or booster medication.

7. Known severe constipation.

8. Disability to comply either cognitively or physically.

The colon capsule endoscopy investigation

Participants who are eligible and mark positive interest in undergoing CCE will be invited to the procedure at one of the out-clinic sites. The procedure will be undertaken using the PillCam Colon 2 (Medtronic, Minneapolis, Minnesota, USA).

To ensure sufficient bowel preparation, participants will have to undergo a bowel preparation procedure before the investigation (table 2).

The participant will receive a bowel preparation kit by mail which is to be completed at home, beginning 72 hours before the CCE. The kit contain polyethylene glycol (PEG) sachets (Movicol, Norgine Danmark A/S, Herlev, Denmark), PEG solutions (MoviPrep, Norgine Danmark A/S, Herlev Denmark) and instructions on how to properly perform the preparation procedures. At the day of ingestion, participants will sign a consent to participate before they swallow the capsule. Afterwards, the participants will be handed Eziclen boosters (Ipsen Pharma, Boulogne-Billancourt, France) and will return home, where they will mix the solution with water to a combined total volume of $1 \mathrm{~L}$ which are ingested at signals 1 to 3 ingesting one glass each time (1/3L). The signals will be given from the receiver. Signal 1 occurs when the capsule reaches the small bowel, signals 2 to 4 will be given every 2 hours after signal 1 . During the investigation the participant is encouraged to be physically active and chew chewing gum. ${ }^{18}$ The participant is instructed to ingest one caffeine tablet and a small snack at signal 3. A suppository, Dulcolax (Sanofi, Paris, France) is to be inserted after signal 4 (table 2). Participants are expected to excrete the capsule 6 to 8 hours after ingestion. After the examination is completed, the

\begin{tabular}{|c|c|c|}
\hline Day & $\begin{array}{l}\text { Colon capsule } \\
\text { endoscopy }\end{array}$ & $\begin{array}{l}\text { Optical } \\
\text { colonoscopy }\end{array}$ \\
\hline-5 & No restrictions & Seedless diet \\
\hline-3 & $\begin{array}{l}2 \times \text { Movicol } 13.8 \mathrm{~g} \text { sachet } \\
\text { Normal diet } \\
2 \mathrm{~L} \text { water }\end{array}$ & Seedless diet \\
\hline-2 & $\begin{array}{l}2 \times \text { Movicol } 13.8 \mathrm{~g} \text { sachet } \\
\text { Normal diet } \\
2 \mathrm{~L} \text { water }\end{array}$ & Seedless diet \\
\hline-1 & $\begin{array}{l}1 \mathrm{~L} \text { MoviPrep+1 L water } \\
\text { Clear liquid diet }\end{array}$ & $\begin{array}{l}1 \mathrm{~L} \text { MoviPrep+1/2 L } \\
\text { water } \\
\text { Seedless diet (until } \\
4 \text { p.m.) } \\
\text { Clear liquid diet } \\
\text { (after } 4 \text { p.m.) }\end{array}$ \\
\hline $\begin{array}{l}0 \\
\text { (procedure } \\
\text { day) }\end{array}$ & $\begin{array}{l}\text { Before capsule intake } \\
1 \mathrm{~L} \text { MoviPrep }+1 \mathrm{~L} \text { water } \\
\text { Clear liquid diet } \\
\text { After capsule intake } \\
\text { Chewing gum } \\
\text { Signal 1: } \\
330 \mathrm{~mL} \text { Eziclen + two to } \\
\text { three large glasses of } \\
\text { water } \\
\text { Signal } 2 \text { : } \\
330 \mathrm{~mL} \text { Eziclen }+ \text { two to } \\
\text { three large glasses of } \\
\text { water } \\
\text { Signal } 3 \text { : } \\
330 \mathrm{~mL} \text { Eziclen }+ \text { two to } \\
\text { three large glasses of } \\
\text { water } \\
1 \times \text { caffeine tablets, } \\
200 \mathrm{mg} \text { and a small fatty } \\
\text { snack, that is, cheese. } \\
\text { Signal } 4 \text { : } \\
\text { Dulcolax } 10 \text { mg, } \\
\text { suppository }\end{array}$ & $\begin{array}{l}1 \mathrm{~L} \text { MoviPrep+1/2 L } \\
\text { water } \\
\text { Clear liquid diet }\end{array}$ \\
\hline
\end{tabular}

participant will return the receiver at the next workday to the out-clinic facility. Staff will upload all endoscopic video material to a secured storage function for diagnostics. Suspected cancer findings will generate an invitation to a diagnostic colonoscopy within 2 days for biopsies, and the patient will subsequently enter the standard treatment protocol. Findings of suspected intermediaterisk or high-risk polyps will generate an invitation to a therapeutic colonoscopy within 7 days. Participants with suspected low-risk polyps will return to the regular screening protocol and will be re-invited 2 years later to submit a FIT. Participants with clean colon will receive an 8-year exclusion from the screening programme.

The CCE investigation is considered complete if the capsule has recorded the anal cushions in participants with acceptable bowel preparation.

In the case of an incomplete CCE investigation for any reason, the participant is referred to colonoscopy. 
An interim analysis will be conducted 6 weeks after initiation, or after inclusion of the first 200 capsule investigations. The analysis will investigate participation and CCE quality in the intervention group. If participation falls below $55 \%$, information material and invitation procedures will be reviewed. If the CCE completion rate is $65 \%$ to $80 \%$, the procedures will be reviewed. If the completion rate is below $65 \%$ in the interim analysis, the trial will be terminated.

\section{Primary outcome}

The primary outcome of this trial is the difference in the number of individuals detected with either intermediaterisk or high-risk adenomas or cancer between the intervention group and the control group. The outcome definitions are based on the Danish CRC screening guidelines for colonoscopy and pathology for maximum comparability. ${ }^{19}$ In addition, a number of secondary outcomes have been established:

1. Acceptability

Participation rates in the intervention group and in the control group will be compared.

2. Rate of major complications

Major complication rate in the intervention group and in the control group.

3. Completion rates

The rates of complete CCE investigations and colonoscopies.

4. Interval CRC rate

CRC detected in the time interval between a complete screening investigation and the next round of screening in the two groups.

5. Total number of colonoscopies avoided due to CCE

The number of colonoscopies that has been avoided due to only low-risk or clean colon findings.

6. Patient-reported outcomes

Four different questionnaires will be administered during the study, the questions detailing the expectations and experiences of the participants in the intervention group.

7. Long-term cancer incidence rates

Cancer incidence rates in the two groups after 2, 5, 10 and 20 years of follow-up.

8. Social inequality

Comparison of participation patterns, findings, experiences and preferences stratified by socioeconomic and demographic characteristics.

9. Applicability and validity of machine learning algorithms in polyp detection, location, characterisation and of bowel cleanliness

Training, implementation and validation of algorithms to improve CCE compared with the manual assessment of the videos. Parameters include polyp detection accuracy, polyp characterisation, bowel preparation assessment and estimation of polyp location and polyp size.

10. Total costs and cost per identified cancer

A cost-effectiveness analysis will be conducted on implementation of CCE.

\section{Data collection and data management}

The CCE video and the accompanying diagnostic report will subsequently be uploaded to the GAstrointestinal Imaging dAtabase (GAIA), where information on all participants will be stored on a secure server. Medical records, questionnaires and diagnostic reports are collected and stored using the software Research Electronic Data Capture (REDCap consortium, Vanderbilt, the Netherlands). For each participant the following information will be collected at baseline:

a. Demographics (eg, age, gender, income, level of education)

b. Lifestyle patterns (eg, smoking, coffee intake, sleep, medicine, physical activity)

c. Perceived stress (Cohen's PSS10) ${ }^{20}$

d. Bowel preparation experiences (eg, compliance, prior experience)

e. Expected and experienced severity of the procedures (eg, discomfort, complications, compliance)

f. Health literacy (HLQ) ${ }^{21}$

g. CCE procedure (eg, completion, excretion, bowel preparation, technical performance)

h. CCE findings (eg, number, location and size of polyps and suspected cancers)

i. Colonoscopy findings (eg, number, location and size of polyps and suspected cancers)

j. Pathology (eg, histology, polyp size, cancer type, cancer size)

Polyps will be matched per location and per size whenever possible. Sensitivity analyses pursuing possible overestimation or underestimation of polyp sizes will be pursued when relevant.

To reduce the risk of bias, the manual data capture process will be subject to several rounds of validation.

\section{Questionnaires}

We will ask participants to fill in a maximum of four questionnaires (Q1, Q2, Q3 and Q4) in a longitudinal fashion following their screening trajectory. In all, the questionnaires include eight modules (figure 2). Q1 is structured in five modules, including demographics, baseline characteristics and behaviour affecting health, participant discomfort estimations and experience of bowel preparation and expected experience of CCE and colonoscopy. Q2 consists of one module investigating behaviour and experience during CCE. Q3 consists of one module investigating the experience of colonoscopy. Q4 investigates health literacy using the Health Literacy Questionnaire (HLQ) developed by Osborne $e t a l^{21}$ translated to Danish by Maindal et al. ${ }^{22}$ Modules not validated prior to this study has been thoroughly qualitatively validated before study start. ${ }^{23}$

\section{Registers}

We will use the Danish Colorectal Cancer Screening Database to access data on administrative screening 


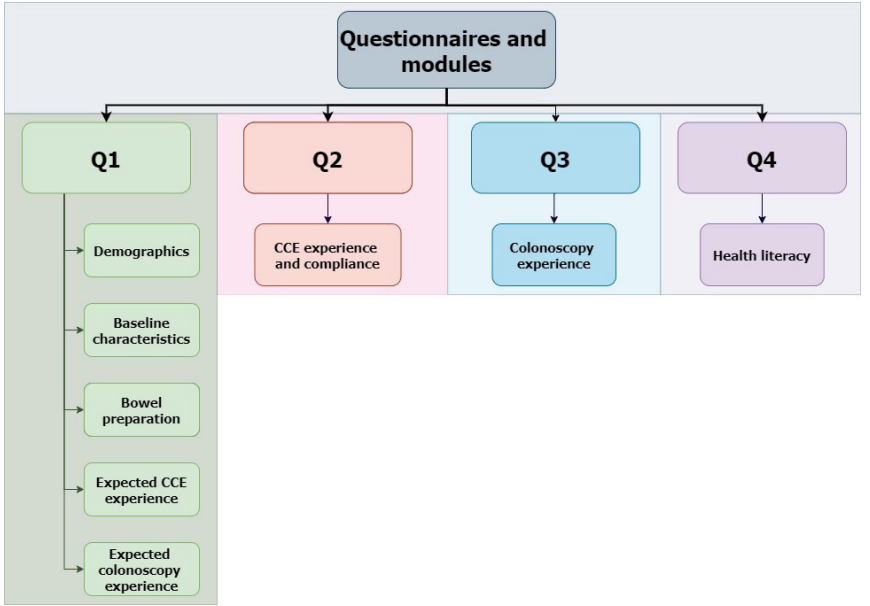

Figure 2 Structure of questionnaire modules. CCE,colon capsule endoscopy.

information not collected via questionnaires or medical records. The register will be important when doing follow-up studies and outcome measures for the control group. In addition, we will collect data from the National Pathology Register which will enable a retrospective validation of the manual entries from the medical records after completion of the study.

Data from the Danish National Patients Register ${ }^{24}$ will be accessed post-study to follow patients in the Danish healthcare system after completion of the trial, thereby enabling monitoring of interval cancers. Using register data provided by Statistics Denmark on demography, ${ }^{25}$ income $^{26}$ and educational level ${ }^{27}$ we will be able to assess individual socioeconomic status.

Data available both in the questionnaires and the registers on topics such as major complications, income and education will be cross-checked to increase the validity of the data.

\section{Statistical analysis}

All numerical variables in the respective studies will be compared using a paired or un-paired t-test. For the categorical variables, a Pearson $X^{2}$ test will be used for comparative purposes. All analysis will be conducted on an intention-to-treat basis. For the primary analysis a multivariate logistic regression model will be used to compare CCE and colonoscopy. Mainly per-patient analyses will be conducted, but in some cases per-polyp analyses will be relevant.

Each substudy will have its own statistical approach. Stata V.16 (StataCorp, Texas, USA), SAS software V.9.4 (SAS institute Inc, SAS 9.4., Cary, North Carolina, USA) and R Statistical Software Package V.3.6.1 (R Core Team, Vienna, Austria) ${ }^{28}$ will be used for data management and analysis.

\section{Sample size}

Based on the findings from our previous studies regarding CCE and the numbers from the Danish colorectal cancer screening programme, we expect $38 \%$ of the control group to have the primary outcome. For the intervention group to have a clinical impact, a $10 \%$ relative corresponding to a $4 \%$ absolute difference between the two groups is needed. Based on this assumption we expect $42 \%$ of the intervention group to have the primary outcome. With a significance level of 0.05 and detection power of 0.8 , a relative difference between the two groups of $10 \%$ will require a minimum of 2352 participants in each group. We anticipate that some will choose colonoscopy over CCE, and we will therefore include 2651 FIT positive participants in the intervention group and 2614 FIT positive participants in the control group.

\section{DISCUSSION}

\section{Clinical considerations}

By offering citizens CCE as an alternative to colonoscopy, we hope to increase the number of significant findings in the intervention group. We also hope to increase participation to screening and we expect to see greater adherence to diagnostic follow-up after a positive FIT. As an expected $50 \%$ of CCE's will not lead to a therapeutic colonoscopy, the demand on endoscopy capacity in hospitals may be reduced significantly. To reduce the need for participants to undergo a potentially unnecessary colonoscopy we will leave behind suspected low-risk polyps detected by CCE. The current screening sensitivity for low-risk polyps has been found to be approximately $7 \%,{ }^{29}$ indicating that most of these polyps may be incidental findings and not the cause of a positive FIT. The polypectomy of low-risk polyps in regular colonoscopy based screening may therefore be considered as redundant and may pose a risk to the patient. ${ }^{30}$ Participants with suspected low-risk polyps will return to biennial screening. By doing so, we expect to reduce the number of participants having to undergo a follow-up colonoscopy by approximately $20 \%$ and we may be able to follow the natural cause of suspected low-risk polyps left behind. Despite these steps to relieve pressure on the hospital sector potentially providing financial surplus, the introduction of CCE as a filter test in CRC screening will not be economically feasible at this moment. However, it is our belief that the gain in diagnostic yield and clinical efficiency provided by the CCE will allow for the omission of elements that are less cost-effective in the current screening programme such as surveillance colonoscopies. Further financial gain from introducing CCE is expected to come from lower capsule prices over time as demands increases. In addition, lower costs of the procedure is expected due to the planned implementation of artificial intelligence algorithms that can optimise the diagnostics process.

\section{Strengths and limitations}

One strength of our multicentre study is that we have a large study population, all randomised from a large geographical area, containing both urban and rural sectors, without considerations to gender, age, race, 
medical history or socioeconomic status. In addition, the nesting of this study in an already implemented screening programme minimise the risk of some of the issues often influencing clinical trials, such as invitation procedures, resource capacities and recruitment of participants.

The use of medical records and register data will allow us to follow participants and controls on a large set of parameters, allowing thorough adjustments for confounding. The same sources will also be used for validation of questionnaire data on relevant covariates that may be subject to response bias (eg, income and education) or recall bias (eg, previous FIT or previous colonoscopy).

This study also has limitations. Data collected from medical records and registers are influenced by the quality of the input from the clinicians in the different hospitals involved. The implementation of a new version of the Danish National Patients Register may impact the quality of the data generated in our study period, which represents a clear limitation. The impact of this implementation is difficult to assess prospectively, but we do not expect it to have a major impact on our findings as missing or faulty data is expected to occur at random in both the intervention group and the control group.

Using data from questionnaires inherently comes with the risk of bias, especially in sensitive questions. However, by cross-validating the answers from the questionnaires, where applicable, with data from medical records and registers we may be able to adjust for the impact of both response and recall bias.

\section{ETHICS AND DISSEMINATION}

This study has been approved by the regional ethics committee under journal number S-20190100. The project has been registered at the Danish Data Protection Agency under journal number 19/29858.

All participants will receive written and verbal information before signing the consent form and the Helsinki declaration is followed. ${ }^{31}$

Correction notice This article has been corrected since it was published. Author name Rasmus Kroijer has been updated.

Acknowledgements We would like to acknowledge the support by OPEN, which has kindly supported the study with technical assistance and tools. We would also like to acknowledge the partners of this study including the Region of Southern Denmark and the hospitals of Esbjerg, Vejle and Aabenraa.

Contributors LK was primarily responsible for drafting the manuscript. UD did part of the drafting. GB is the principal investigator and sketched the overall concept of the manuscript. GB, LK, UD, MK-L, A-LVH, EZ-N, MKT, RK and TB-M all contributed to the development of the trial with separate areas of focus ranging from the clinical (GB, EZ-N, MKT, MK-L, RK, TB-M) to the legal (LK, MK-L, MKT, GB, EZ-N) and to the scientific aspects, including data handling, capture and storage (GB, LK, UD, MKT, MK-L, A-LVH). GB drafted the protocol on which this manuscript is based, assisted by MK-L, MKT and RK.

Funding This work was supported by Aage and Johanne Louis-Hansen's Fond (grant 17-2B-1409), OUH's innovation fund (grant: R75-A3392), Medtronic Research Foundation (grant ERP 2018-11151), the Danish Cancer Society (grant R100-A6747) and the Excellence Centre in the Region of Southern Denmark (grant 18/48426).
Competing interests GB is financially supported by Medtronic but the company does not influence any scientific processes, and no investigator will receive any personal benefits.

Patient consent for publication Not required.

Provenance and peer review Not commissioned; externally peer reviewed.

Data availability statement Data will be available upon reasonable request.

Open access This is an open access article distributed in accordance with the Creative Commons Attribution Non Commercial (CC BY-NC 4.0) license, which permits others to distribute, remix, adapt, build upon this work non-commercially, and license their derivative works on different terms, provided the original work is properly cited, appropriate credit is given, any changes made indicated, and the use is non-commercial. See: http://creativecommons.org/licenses/by-nc/4.0/.

\section{ORCID iDs}

Lasse Kaalby http://orcid.org/0000-0002-6721-3604

Ulrik Deding http://orcid.org/0000-0002-8263-2989

Morten Kobaek-Larsen http://orcid.org/0000-0002-5097-9283

Anne-Line Volden Havshoi http://orcid.org/0000-0003-2376-7389

Erik Zimmermann-Nielsen http://orcid.org/0000-0002-7385-4969

Marianne Kirstine Thygesen http://orcid.org/0000-0002-1811-7405

Rasmus Kroijer http://orcid.org/0000-0003-4358-7916

Thomas Bjørsum-Meyer http://orcid.org/0000-0001-5253-0802

Gunnar Baatrup http://orcid.org/0000-0003-0300-5766

\section{REFERENCES}

1 Navarro M, Nicolas A, Ferrandez A, et al. Colorectal cancer population screening programs worldwide in 2016: an update. World J Gastroenterol 2017;23:3632-42.

2 Kronborg O, Fenger C, Olsen J, et al. Randomised study of screening for colorectal cancer with faecal-occult-blood test. Lancet 1996;348:1467-71.

3 Shaukat A, Mongin SJ, Geisser MS, et al. Long-term mortality after screening for colorectal cancer. N Engl J Med 2013;369:1106-14.

4 Mandel JS, Bond JH, Church TR, et al. Reducing mortality from colorectal cancer by screening for fecal occult blood. minnesota colon cancer control study. N Engl J Med 1993;328:1365-71.

5 Scholefield JH, Moss SM, Mangham CM, et al. Nottingham trial of faecal occult blood testing for colorectal cancer: a 20 -year followup. Gut 2012;61:1036-40.

6 Hardcastle JD, Chamberlain JO, Robinson MH, et al. Randomised controlled trial of faecal-occult-blood screening for colorectal cancer. Lancet 1996;348:1472-7.

7 Deding U, Henig AS, Salling A, et al. Sociodemographic predictors of participation in colorectal cancer screening. Int $J$ Colorectal Dis 2017;32:1117-24.

8 Tamrkræftscreeningsdatabase SfD. Dansk tarmkræftscreeningsdatabase arsrapport 2017 nationale prævalens screeningsrunde [Danish colorectal cancer screening databse annual report 2017 national prevalence screening round], 2018.

9 Deding U, Henig AS, Hindersson P, et al. Determinants of nonparticipation in colon examination following positive stool sample in colorectal cancer screening. Eur J Public Health 2019;29:1118-24.

10 Thomsen MK, Njor SH, Rasmussen M, et al. Validity of data in the Danish colorectal cancer screening database. Clin Epidemiol 2017;9:105-11

11 Mikkelsen EM, Thomsen MK, Tybjerg J, et al. Colonoscopy-related complications in a nationwide immunochemical fecal occult blood test-based colorectal cancer screening program. Clin Epidemiol 2018;10:1649-55.

12 Groth S, Krause H, Behrendt R, et al. Capsule colonoscopy increases uptake of colorectal cancer screening. BMC Gastroenterol 2012;12:80

13 Thygesen MK, Baatrup G, Petersen C, et al. Screening individuals' experiences of colonoscopy and colon capsule endoscopy; a mixed methods study. Acta Oncol 2019;58:S71-6.

14 Kobaek-Larsen M, Kroijer R, Dyrvig A-K, et al. Back-to-back colon capsule endoscopy and optical colonoscopy in colorectal cancer screening individuals. Colorectal Dis 2018;20:479-85.

15 Holleran G, Leen R, O'Morain C, et al. Colon capsule endoscopy as possible filter test for colonoscopy selection in a screening population with positive fecal immunology. Endoscopy 2014;46:473-8.

16 Njor SH, Friis-Hansen L, Andersen B, et al. Three years of colorectal cancer screening in Denmark. Cancer Epidemiol 2018;57:39-44. 
17 Zelen M. A new design for randomized clinical trials. N Engl J Med 1979;300:1242-5.

18 Buijs MM, Kobaek-Larsen M, Kaalby L, et al. Can coffee or chewing gum decrease transit times in colon capsule endoscopy? A randomized controlled trial. BMC Gastroenterol 2018;18:95.

19 Den tværregionale implementeringsgruppe vedr. Screenings- og adenomkontrol program for tyk-og endetarmskræft, guidelines for koloskopi og patologi [Screening- and adenomacontrol program for colorectal cancer, guidelines for colonoscopy and pathology]. Danske Regioner, 2014.

20 Cohen S. Perceived stress in a probability sample of the United States. the social psychology of health. Thousand Oaks, CA, US: Sage Publications, Inc, 1988: 31-67.

21 Osborne RH, Batterham RW, Elsworth GR, et al. The grounded psychometric development and initial validation of the health literacy questionnaire (HLQ). BMC Public Health 2013;13:658-58.

22 Maindal HT, Kayser L, Norgaard O, et al. Cultural adaptation and validation of the health literacy questionnaire (HLQ): robust ninedimension Danish language confirmatory factor model. Springerplus 2016;5:1232-32
23 Power A, Lemay J-F, Cooke S. Justify your answer: the role of written think aloud in script concordance testing. Teach Learn Med 2017;29:59-67.

24 Lynge E, Sandegaard JL, Rebolj M. The Danish national patient register. Scand J Public Health 2011;39:30-3.

25 Pedersen CB. The Danish civil registration system. Scand J Public Health 2011;39:22-5.

26 Baadsgaard M, Quitzau J. Danish registers on personal income and transfer payments. Scand J Public Health 2011;39:103-5.

27 Jensen VM, Rasmussen AW. Danish education registers. Scand J Public Health 2011;39:91-4.

28 R Core Team. R: a language and environment for statistical computing, 2019.

29 Morikawa T, Kato J, Yamaji Y, et al. Sensitivity of immunochemical fecal occult blood test to small colorectal adenomas. Am J Gastroenterol 2007;102:2259-64.

30 Kandel P, Wallace MB. Should we resect and discard low risk diminutive colon polyps. Clin Endosc 2019;52:239-46.

31 World Medical Association. World medical association declaration of Helsinki: ethical principles for medical research involving human subjects. JAMA 2013;310:2191-4. 